\title{
Nanoencapsulation of n-Octadecane Phase Change Material with Polyaniline Shell for Thermal Energy Storage
}

\author{
Shuang Fan ${ }^{1, a}$, Hongyi Gao ${ }^{1, b}$, Qiming $\mathrm{Xu}^{1, \mathrm{c}}$, Huanqiu He ${ }^{1, \mathrm{~d}}$, Lili $\mathrm{Lu}^{1, \mathrm{e}}$, Junyao \\ Shen $^{1, f}, \mathrm{Yu} \mathrm{Xiao}^{1, g}$ and Li Tan ${ }^{2, \mathrm{~h} *}$ \\ ${ }^{1}$ Department of Materials Science and Engineering, University of Science and Technology Beijing, No \\ 30 Xueyuan Road, Haidian District, Beijing, 100083, China \\ 2 Institute for Advanced Materials and Technology, University of Science and Technology Beijing, \\ No 30 Xueyuan Road, Haidian District, Beijing, 100083, China \\ a15910751786@126.com, b15210600103@163.com, '857467411@qq.com, d497891518@qq.com, \\ e438097705@qq.com, ${ }^{f} 625003680 @ q q . c o m,{ }^{9} 1049982139 @ q q . c o m,{ }^{h}$ tljw060606@163.com
}

Keywords: Nanoencapsulated n-octadecane, Polyaniline, Phase change performance.

Abstract. Nanoencapsulated n-octadecane with polyaniline shell as composite thermal energy storage material was synthesized using emulsion polymerization method. In this composite material, the n-octadecane was employed as the core material that is the latent heat storage phase change material (PCM), and the polyaniline acted as the shell material which can avoided the leakage of the melted n-octadecane. The scanning electronic microscope (SEM) and transmission electron microscope (TEM) were used to determine microstructure of nanoencapsulated n-octadecane with polyaniline shell. The fourier transformation infrared spectroscope (FT-IR) and X-ray diffraction (XRD) were employed to determine chemical structure of these composites. The thermal properties were shown by the differential scanning calorimeter (DSC).

\section{Introduction}

The phase change material (PCM) application in latent thermal storage energy plays a crucial role in thermal energy storage technology, since the phase change material can absorb, store or release large amounts of latent heat over phase changes[1, 2, 3]. The most important advantage of PCM is high energy storage densities at almost constant temperature. Thus, tremendous efforts of PCM have been developed and numerous improvement have been achieved. Specially, the n-octadecane was the well-known PCMs due to applied to textiles with a suitable melting temperature. In addition, the n-octadecane are nontoxic, non-corrosive, chemically inert, easily obtained and have no unpleasant odor [4, 5]. Obviously, this phase change temperature of n-octadecane is comfortable for the human body and it possess the advantages of high latent heat $(227 \mathrm{~J} / \mathrm{g})$. Then, the nanoencapsulation technique can successfully avoid the leakage of n-octadecane from their location when the phase change occurs.

Polyaniline (PANI) not only possess good environment stability, but also can be made in a variety of morphology [6]. Thus, it is worthwhile to employ PANI as supporting material to prepare composite PCMs. In this study, we proposed a facile method to fabricate the n-octadecane/PANI nanocapsules with different ratios of n-octadecane to polyaniline. The PANI was selected as shell material in order to fabricate environmentally friendly the nanoencapsulation process in an aqueous system.

\section{Experimental}

\section{Materials}

All reagents were of a nalytical grade and were obtained commercially. A aniline was distilled under reduced pressure prior to use. All other reagents were used as received without further purification. Deionized water was used throughout the experimental process. 


\section{Preparation of nanoencapsulated PCMs}

A series of nanoencapsulated PCMs were prepared as follows. $0.12 \mathrm{~g}$ of styrene maleic anhydride (SMA) and $0.1 \mathrm{~g} \mathrm{NaOH}$ were mixed with $50 \mathrm{~mL}$ water. The mixture was stirred for $0.5 \mathrm{~h}$ at $80{ }^{\circ} \mathrm{C}$ to form the transparent solution. The transparent solution were mixed and followed by adding Aniline and n-octadecane with desired amounts, $1.5 / 1 \mathrm{~g}, 1.25 / 1.25 \mathrm{~g}, 1 / 1.5 \mathrm{~g}$, that are named as S1, S2, S3, respectively. After being pre-emulsified by a homogenizer at a rate of $10000 \mathrm{rpm}$ for $10 \mathrm{~min}$, miniemulsification was continued by ultrasonicating the mixture for $10 \mathrm{~min}$ at $70 \%$ amplitude at $45{ }^{\circ} \mathrm{C}$. Then, ammonium persulfate (APS) was dissolved in $10 \mathrm{~mL}$ of water and dropped into the mixture to initiate the polymerization. The molar ratio of aniline and APS should be kept to be $1: 1$. The mixture was stirred for $12 \mathrm{~h}$ at $0-5{ }^{\circ} \mathrm{C}$ and the polymerization was completed.

\section{Characterization}

The surface morphology and microstructures of the nanoencapsulated PCMs were revealed using a scanning electron microscope (SEM, ZEISS SUPRA55) (FE-SEM) and a transmission electron microscope (TEM, JEOL JEM-100CX II ) operating at $200 \mathrm{kV}$, respectively. IR spectra were recorded with a Nicolet 6700 Fourier spectrometer using $\mathrm{KBr}$ pellet (400- $\left.4000 \mathrm{~cm}^{-1}\right)$. And, the wide angle X-ray diffraction (XRD) experiments were carried out on a X-ray powder diffraction (XRD, M21X) with $\mathrm{Cu}$ Ka radiation $(40 \mathrm{kV}$, $150 \mathrm{~mA}, \lambda=1.5406 \AA$ ). The thermal energy storage properties of n-octadecane and the composite PCMs were characterized by differential scanning calorimetry (DSC, Mettler DSC 822e) from -5 to $60{ }^{\circ} \mathrm{C}$ with the heating rate of $5{ }^{\circ} \mathrm{C} / \mathrm{min}$ in nitrogen atmosphere.

\section{Results and discussion}

\section{Morphology characterization of the n-octadecane/PANI nanocapsules}

Fig. 1 presents SEM and TEM photographs of S1, S2 and S3. The n-octadecane was encapsulated in the shells of the PANI. The PANI shells provided the mechanical strength for the pure n-octadecane and prevented the leakage of the melted n-octadecane. As shown Fig. 1a-c, that morphologies of S1, S2 and S3 are spherical with nano-sized of 500-700 nm. It is seen from Fig. 1d-f that microstructures of S1, S2 and S3 are core-shell structure.

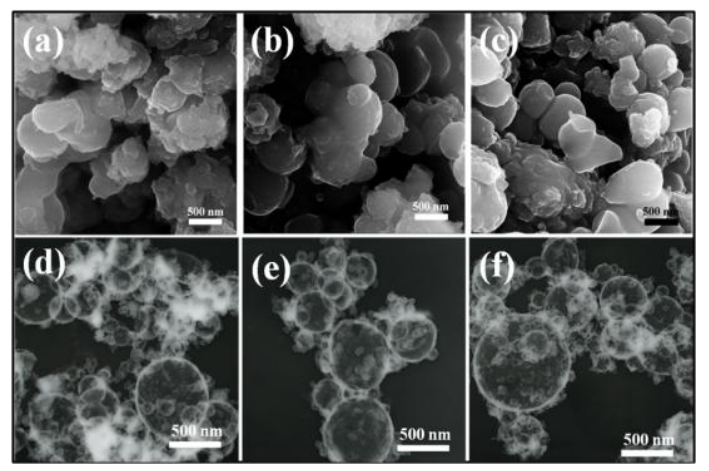

Fig.1. SEM and TEM photographs of S1 (a) and (d), S2 (b) and (e), S3 (c) and (f).

The FT-IR spectra of PANI, n-octadecane and n-octadecane/PANI nanocapsules are displayed in Fig. 2. The peak of n-octadecane can be ascribed as follows. The band appeared at $2853 \mathrm{~cm}^{-1}$ and $2953 \mathrm{~cm}^{-1}$, which is ascribed to the absorption of the $\mathrm{C}-\mathrm{H}$ stretching vibration of methylene and methyl groups, and the absorption peak at $1466 \mathrm{~cm}^{-1}$ and $721 \mathrm{~cm}^{-1}$ are associated with $\mathrm{C}-\mathrm{H}$ bending vibration of methyl groups and $\mathrm{C}-\mathrm{H}$ in-plane rocking vibration of methylene groups. In the PANI spectrum, the absorption peaks at 1568 $\mathrm{cm}^{-1}$ and $1486 \mathrm{~cm}^{-1}$ correspond to the $\mathrm{C}-\mathrm{C}$ stretching vibration of quinonoid and benzene rings, respectively. The absorption peak at $1297 \mathrm{~cm}^{-1}$ is related to the $\mathrm{C}-\mathrm{N}$ stretching vibration of the secondary aromatic amine. 
The spectra of n-octadecane/PANI nanocapsules shows clearly spectra of both characteristic peaks of n-octadecane and PANI, which indicate that clearly n-octadecane exists nanocapsules. What is more, no new peak appeared, indicating no chemical interaction occurred among PANI and n-octadecane.

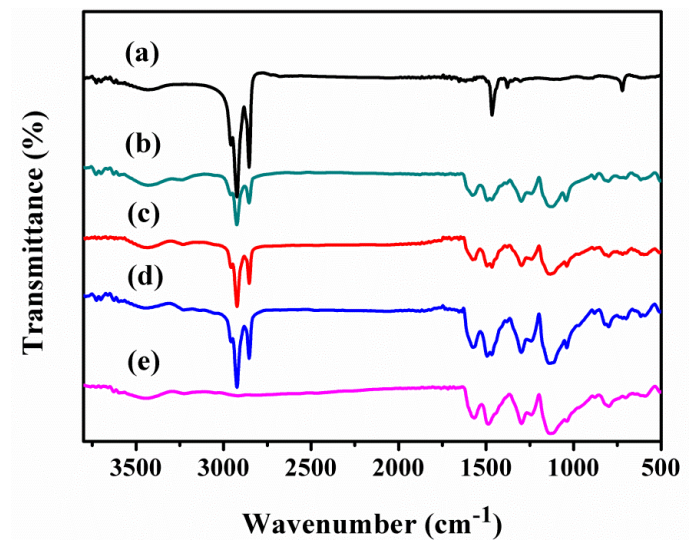

Fig. 2. FT-IR spectra of the (a) n-octadecane, (b) S1, (c) S2, (d) S3 and (e) PANI.

Fig. 3. shows the XRD patterns of the n-octadecane, S3, S2 and S1. The XRD pattern of PANI in S1, S2 and S3 agrees with Ref [7]. The characteristic peaks of $20^{\circ}$ and $25^{\circ}$ are corresponding to (020) and (200) crystal planes of PANI. The microcapsule samples S3 mainly revealed the characteristic peaks of n-octadecane coordinate with the strong diffraction intensity of crystalline n-octadecane. The characteristic peaks at $19.24^{\circ}, 19.81^{\circ}$ and $23.31^{\circ}$ are assigned to (l 100$),\left(\begin{array}{lll}0 & 1 & 1\end{array}\right)$, and $\left(\begin{array}{lll}1 & 0 & 5\end{array}\right)$ which are related to the crystal of $n$-octadecane. Therefore, the XRD pattern not only comfirm the existence of $n$-octadecane inside nanocapsules, but also demonstrate the good crystallinity of n-octadecane within the nanocapsules.

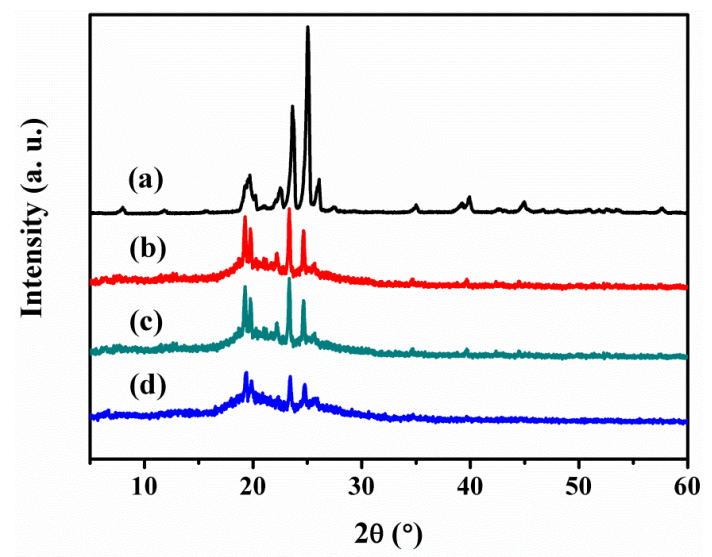

Fig. 3. XRD patterns of the (a) n-octadecane, (b) S3, (c) S2 and (d) S1.

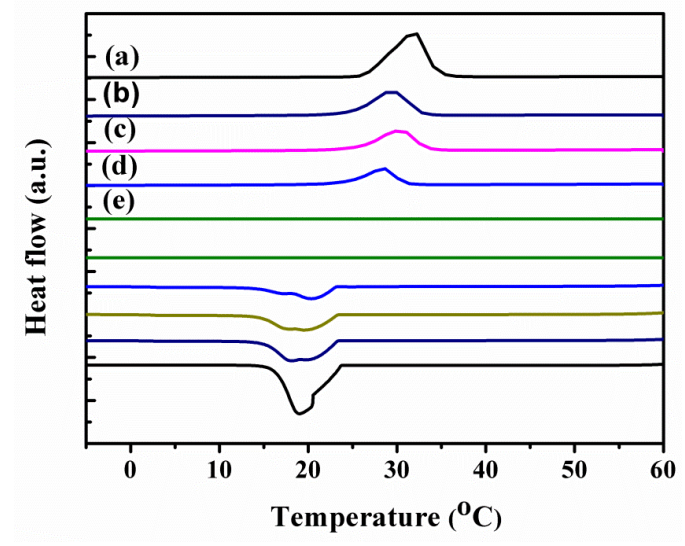

Fig. 4. The melting and solidifying DSC curves of (a) n-octadecane, (b) S3, (c) S2, (d) S1 and (e) PANI. 
Fig.4 shows the DSC curves of pristine n-octadecane, PANI and S1, S2, S3 fabricated at different monomer/PCM ratios, respectively. The phase transition temperature and latent storage capacity were shown in Table 1. By considering these results, it can be clearly stated that S1, S2 and S3 exhibit very stable phase change behaviors in their heating and cooling periods. The pristine n-octadecane has also melting peak at $29.5^{\circ} \mathrm{C}$, and freezing peak at $21.1^{\circ} \mathrm{C}$. The different composite PCMs, namely S1, S2 and S3 showed melting peak at $27.5,27.9$ and $28.3{ }^{\circ} \mathrm{C}$, and freezing peak at $20.9,20.3$ and $21.2{ }^{\circ} \mathrm{C}$, respectively. The sub-cooling degree of S1, S2 and S3 were reduced which can provides an important advantage for composite PCMs. With the increase of the feed amount of n-octadecane, the $\Delta H_{m}$ of S1, S2 and S3 will increases. In particular, $\Delta \mathrm{H}_{\mathrm{m}}$ of $\mathrm{S} 3$ are $105.4 \mathrm{~J} / \mathrm{g}$ indicating a relatively best energy storage capacity.

Table 1. DSC data of the n-octadecane, S1, S2 and S3.

\begin{tabular}{lllll}
\hline Sample & $\begin{array}{l}\mathrm{T}_{\mathrm{pm}} \\
\left({ }^{\circ} \mathrm{C}\right)\end{array}$ & $\begin{array}{l}\Delta \mathrm{H}_{\mathrm{m}} \\
\left(\mathrm{Jg}^{-1}\right)\end{array}$ & $\begin{array}{l}\mathrm{T}_{\mathrm{pc}} \\
\left({ }^{\circ} \mathrm{C}\right)\end{array}$ & $\begin{array}{l}\Delta \mathrm{H}_{\mathrm{c}} \\
\left(\mathrm{Jg}^{-1}\right)\end{array}$ \\
\hline PANI & - & - & - & - \\
S1 & 27.5 & 63.3 & 20.9 & 67.4 \\
S2 & 27.9 & 92.4 & 20.3 & 94.5 \\
S3 & 28.3 & 105.4 & 21.2 & 108.4 \\
n-octadecane & 29.5 & 227.4 & 21.1 & 233.7 \\
\hline
\end{tabular}

Note: $T_{\mathrm{pm}}$, Peak temperature on DSC heating curve; $\mathrm{T}_{\mathrm{pc}}$, Peak temperature on DSC cooling curve.

\section{Conclusions}

In summary, the spherical nanocapsules of PANI-shelled n-octadecane were prepared. These nanocapsules have relatively higher energy storage capacity of 63.3-105.4 J/g and a proper mean diameter of 500-700 nm within the scale desirable for textile coatings.

\section{Acknowledgments}

We thank the Fundamental Research Funds for the Central Universities (FRF-TP-15-053A2) and the National Natural Science Foundation of China (No. 51436001) for support.

\section{References}

[1] F. Agyenim, N. Hewitt, P. Eames, Renew. Sust. Energ. Rev., 14 (2010) 615-628.

[2] J. Jeon, J.H. Lee, J. Seo, J. therm. Anal. Calorim., 111 (2013) 279-288.

[3] G. Li, Y. Hwang, R. Radermacher, 51 (2013) 1-17.

[4] H. Zhang, S. Sun, X. Wang, Colloid. Surf. A, Physicochem. Eng. Asp., 389 (2011) 104-117.

[5] H. Zhang, X. Wang, D. Wu, J. colloid interf. Sci., 343 (2010) 246-255.

[6] J.L. Zeng, F.R. Zhu, S.B. Yu, Sol. Energ. Mat. Sol. C., 114 (2013) 136-140.

[7] J. Yin, X. Zhao, X. Xia, Polymer, 49 (2008) 4413-4419. 